\title{
Age of acquisition effects in picture naming: evidence for a lexical-semantic competition hypothesis
}

\author{
Eva Belke $^{\mathrm{a}, *}$, Marc Brysbaert ${ }^{\mathrm{b}}$, Antje S. Meyer ${ }^{\mathrm{c}}$, Mandy Ghyselinck ${ }^{\mathrm{d}}$ \\ ${ }^{a}$ Department of Psychology, School of Life and Health Sciences, Aston University, \\ Aston Triangle, Birmingham B4 7ET, UK \\ ${ }^{\mathrm{b}}$ Department of Psychology, Royal Holloway, University of London, UK \\ ${ }^{\mathrm{c}}$ Department of Psychology, University of Birmingham, UK \\ ${ }^{\mathrm{d}}$ Department of Experimental Psychology, Ghent University, Belgium
}

Received 11 October 2004; accepted 30 November 2004

\begin{abstract}
In many tasks the effects of frequency and age of acquisition (AoA) on reaction latencies are similar in size. However, in picture naming the AoA-effect is often significantly larger than expected on the basis of the frequency-effect. Previous explanations of this frequency-independent AoA-effect have attributed it to the organisation of the semantic system or to the way phonological word forms are stored in the mental lexicon. Using a semantic blocking paradigm, we show that semantic context effects on naming latencies are more pronounced for late-acquired than for early-acquired words. This interaction between AoA and naming context is likely to arise during lexical-semantic encoding, which we put forward as the locus for the frequency-independent AoA-effect.
\end{abstract}

(C) 2005 Elsevier B.V. All rights reserved.

Keywords: Age-of-acquisition; Picture naming; Lexical retrieval; Semantic context

Word processing times depend not only on the frequency with which the words occur in texts, but also on the age at which they were acquired (age-of-acquisition, AoA). Words that were acquired early in life are responded to faster than late-acquired words of the same frequency (Morrison \& Ellis, 1995). In word naming and lexical decision, the effects

\footnotetext{
* Corresponding author. Tel.: +44 121204 4079; fax: +44 1213593257.

E-mail address: e.belke@bham.ac.uk (E. Belke).
} 
of AoA and frequency tend to be highly correlated and of similar size, supporting the view that both effects are the result of a single underlying learning principle (e.g. Ellis \& Lambon Ralph, 2000; Lewis, Gerhand, \& Ellis, 2001; for a review see Brysbaert \& Ghyselinck, in press). In picture naming, however, the AoA-effect tends to be much larger than predicted on the basis of the frequency-effect (e.g. Barry, Hirsh, Johnston, \& Williams, 2001; Bates, Burani, D'Amico, \& Barca, 2001).

The present research concerns the locus of the frequency-independent effect of AoA in picture naming. Two reasons why an AoA effect in picture naming may occur without an accompanying frequency effect have been proposed: The phonological completeness hypothesis (Brown \& Watson, 1987) states that early learned words are stored holistically in the speech output lexicon, whereas later acquired words are stored in a fragmented way and must be assembled anew each time they are produced. The semantic hypothesis (Brysbaert, Van Wijnendaele, \& De Deyne, 2000) says that the order of acquisition determines the speed with which the semantic representations of concepts can be activated, with early acquired concepts being more accessible than late-acquired concepts. Both hypotheses have been called into question, however. Monaghan and Ellis (2002) reported that participants were not faster to segment late-acquired words than earlyacquired words, which argues against the phonological completeness hypothesis (see also Izura \& Ellis, 2004, for further evidence against this hypothesis). In semantic decision tasks Brysbaert and Ghyselinck (in press) failed to find an AoA-effect that was stronger than could be expected on the basis of the frequency-effect, refuting the semantic hypothesis.

In our research, we examined the hypothesis that the frequency-independent AoAeffect seen in picture naming arose during the mapping from conceptual knowledge onto linguistic units. Picture naming necessarily involves a lexical-semantic encoding process during which one lexical unit, a so-called lemma (see Levelt, Roelofs, \& Meyer, 1999), is selected from a set of conceptually similar candidates (see also Caramazza, 1997; Dell, Schwartz, Martin, Saffran, \& Gagnon, 1997, for further discussion of the properties of lexical units and the selection process). During word naming, readers must retrieve the phonological form corresponding to the written input. Lexical-semantic information about the word will be activated during this process, but lemma selection is not a prerequisite for response initiation (e.g. Coltheart, Rastle, Perry, Langdon, \& Ziegler, 2001; Roelofs, 2004).

We hypothesized that the frequency-independent AoA-effect in picture naming might arise because the lemmas of early-acquired words are stronger competitors than those of late-acquired words. To test this hypothesis, we used a semantic blocking paradigm: Participants repeatedly named sets of objects from the same or from different semantic categories and their naming latencies were recorded. In the blocking paradigm, naming latencies are longer for homogeneous (same-category) than for heterogeneous sets (e.g. Damian, Vigliocco, \& Levelt, 2001; Kroll \& Stewart, 1994). Belke, Meyer, and Damian (in press) argued that the items within homogeneous lists activated each other via shared category and feature nodes at the conceptual level and therefore compete more vigorously during lexical-semantic encoding than the items in heterogeneous lists. In line with this account, they showed that the semantic context effect generalized to new, previously unnamed members of the same semantic category. This suggests that 
in the homogeneous condition not only the lemmas from the list become highly activated but, via the connections to shared category and feature nodes, also other lemmas that belong to the same category.

We predicted that the semantic context effect should be stronger for homogeneous sets of late-acquired words than for homogeneous sets of early-acquired words. This interaction should arise because early-acquired lemmas should be more powerful extralist competitors to late-acquired lemmas in a homogeneous naming context than vice versa. For instance, if speakers repeatedly name four flowers, the lemmas of the earlyacquired words "daisy" and "rose" are stronger competitors (e.g. by virtue of being faster to activate) for the list of late acquired names "orchid, crocus, bluebell, lavender" than the lemmas "orchid" and "bluebell" are for the list "daisy, buttercup, daffodil, rose".

To test this prediction, we crossed semantic context (homogeneous, heterogeneous) with AoA (early, late). Two groups of participants were tested: One named pictures of objects and the other read aloud the object names. The latter group was included to establish that the AoA-effect would be stronger in picture naming than in word reading, and that the interaction of AoA and semantic context would only arise during object naming but not during word reading.

\section{Method}

\subsection{Participants}

Thirty-six undergraduate students from the University of Birmingham participated in the experiment in exchange for course credits or payment. Twenty participants carried out the picture naming task and sixteen the word reading task.

\subsection{Materials}

For the picture naming task, we selected 16 objects with early-acquired names and 16 with late-acquired names using the picture gallery provided by Snodgrass and Vanderwart (1980) and by Morrison, Chappell, and Ellis (1997). Four homogeneous and four heterogeneous sets each were created of the early- and the late-acquired sets (see Appendix). Each homogeneous set contained four items from the same category, whereas each heterogeneous set included one item from each of the four semantic categories. The early and late acquired sets were matched as tightly as possible on various lexical variables (see Table 1). In an independent study with 16 participants, the pictures in homogeneous sets were rated as slightly more similar in their visual features (early: 2.15 , late: 2.36 on a scale from 1 to 5 ) than those in heterogeneous sets (early: 1.53 , late: 1.46 ). These ratings are in the same range as those reported by Damian, Vigliocco, and Levelt (2001) and differ markedly from those for highly visually similar filler pairs (4.46).

The pictures were presented centred on the screen as black line drawings on a white background. They were fit into frames sized $6^{\circ} \times 6^{\circ}$ (at $60 \mathrm{~cm}$ viewing distance). In the word naming experiment, the object names were presented in upper case, using Arial size 36 type font. 
Table 1

Characteristics of the stimuli (from Morrison et al., 1997)

\begin{tabular}{|c|c|c|c|c|c|c|c|c|}
\hline & \multicolumn{2}{|c|}{ Fruit \& vegetables } & \multicolumn{2}{|c|}{ Animals } & \multicolumn{2}{|c|}{ Tools } & \multicolumn{2}{|c|}{ Clothing } \\
\hline & Early & Late & Early & Late & Early & Late & Early & Late \\
\hline AoA & 32.9 & 83.0 & 23.5 & 86.0 & 31.0 & 113.9 & 31.1 & 104.0 \\
\hline Ima. & 6.3 & 5.9 & 6.5 & 6.0 & 6.4 & 5.8 & 6.2 & 5.6 \\
\hline Freq. & 0.97 & 0.96 & 1.12 & 0.93 & 0.71 & 0.3 & 1.44 & 0.49 \\
\hline Fam. & 3.8 & 3.2 & 2.5 & 2.0 & 3.0 & 2.3 & 4.3 & 2.9 \\
\hline $\begin{array}{l}\text { Vis. } \\
\text { Compl. }\end{array}$ & 2.1 & 2.5 & 3.2 & 3.4 & 2.3 & 2.4 & 3.0 & 3.1 \\
\hline $\begin{array}{l}\text { Name } \\
\text { Agr. }\end{array}$ & 0.9 & 0.9 & 1.0 & 0.8 & 0.9 & 0.8 & 0.9 & 0.8 \\
\hline $\begin{array}{l}\text { Picture } \\
\text { Agr. }\end{array}$ & 4.5 & 4.3 & 4.6 & 4.4 & 4.7 & 4.5 & 4.6 & 4.4 \\
\hline $\begin{array}{l}\text { Pho- } \\
\text { nemes }\end{array}$ & 4.8 & 4.5 & 4.5 & 4.5 & 5.3 & 4.5 & 4.3 & 4.3 \\
\hline Syllables & 2.0 & 2.0 & 1.8 & 2.0 & 2.0 & 1.8 & 2.0 & 2.0 \\
\hline $\begin{array}{l}\text { Word } \\
\text { Length }\end{array}$ & 5.5 & 6.0 & 5.0 & 5.8 & 7.5 & 6.0 & 5.8 & 6.5 \\
\hline
\end{tabular}

Age of Acquisition (AoA75, i.e. the age (in months) at which $75 \%$ of the children could name the picture), Imageability, Frequency $\left(\log _{10} /\right.$ million; Celex), Familiarity, Visual Complexity, Name Agreement, Picture-Name Agreement, Number of Phonemes, Number of Syllables and Word Length in letters for early- and late-acquired words in each category.

\subsection{Apparatus}

The experiment was controlled by the Nijmegen Experimental SetUp (NESU). Reaction times were registered using a voice key (HASOMED Nesu-Box 2) and a Sony ECM-MS907 microphone.

\subsection{Design}

The variables AoA (early, late) and semantic context (homogeneous, heterogeneous) were varied within participants and the task (picture vs. word naming) between participants. Six presentation cycles were created from each homogeneous and each heterogeneous set and concatenated to 24-item stimulus lists. The eight early- and the eight late-acquired stimulus lists were presented in separate test sessions, the order of which was counterbalanced across participants. Within each session, homogeneous and heterogeneous lists were tested in alternation, with half of the participants beginning with a homogeneous and half with a heterogeneous list. Each experimental session began with a practice block of 16 trials, in which all items were shown once in random order, followed by two blocks of experimental stimuli, each including two homogeneous and two heterogeneous lists.

\subsection{Procedure}

Participants read the instruction and subsequently completed the two experimental sessions, with a short break in-between. Prior to each experimental session, the participants 
in the picture-naming group studied a booklet showing the pictures used in the upcoming session and the names that should be used. The participants were asked to use bare nouns (e.g. "frog"). Each trial started with the presentation of a fixation point for $500 \mathrm{~ms}$, followed by a 100-ms blank interval, the presentation of the target for $1100 \mathrm{~ms}$ and another blank interval of $800 \mathrm{~ms}$.

\section{Results}

We performed analyses of variance (ANOVAs) on the proportions of correct responses and the reaction times obtained on valid trials using participants as a random factor (see Belke et al., in press).

\subsection{Picture naming}

$3.8 \%$ of the trials were excluded because of naming errors, hesitations or technical errors. The error rate was higher in the homogeneous than in the heterogeneous context $(4.3 \%$ vs. $3.3 \% ; F(1,19)=5.73, P<.05)$ and it was lower in the early-acquired than in the late-acquired sets $(3.1 \%$ vs. $4.5 \% ; F(1,19)=6.94, P<.05)$. The AoA effect was most pronounced on the first presentation cycle, yielding a significant interaction of presentation cycle and AoA $(F(5,95)=3.99, P<.01)$ but no main effect of presentation cycle. When the first presentation cycle was excluded, only the main effect of context remained significant $(F(1,19)=7.73, P<.05)$.

Fig. 1(a) shows the average naming latencies on correct trials broken down by AoA, context, and presentation cycle. There was no effect of context on the first presentation cycle. This is because the semantic context is only established during this cycle. As in earlier studies (Belke et al., in press; Damian et al., 2001), the data from the first presentation cycle were excluded from the statistical analyses concerning the semantic context effect.

For the remaining presentation cycles, we obtained significant main effects of presentation cycle $(F(4,76)=3.55, P<.05)$, with latencies decreasing slightly across the repetitions of the materials, of context $(F(1,19)=46.95, P<.001)$, AoA $(F(1,19)=83.97$, $P<.001)$ and, most importantly, a significant interaction of context and AoA $(F(1,19)$ $=5.04, P<.05)$. As predicted, the context effect was larger for late-acquired picture names $(26 \mathrm{~ms} ; F(1,19)=19.76, P<.001)$ than for early-acquired names $(15 \mathrm{~ms}$; $F(1,19)=41.7, P<.001)$.

As seen in Table 1, the stimulus lists were not perfectly matched for word frequency. However, stepwise multiple regression analyses confirmed that latency differences between the AoA-sets arose primarily from the AoA-differences between the items. When entered first, AoA accounted for $56.4 \%$ of the variance in the item means $(F(1,30)=38.81$, $P<.001$ ); adding frequency only increased the explained variance by $0.6 \%$. When the two predictors were entered in reverse order, AoA significantly increased the explained variance from $22.8 \%$ (frequency-only, $F(1,30)=8.85, P<.05)$ to $57 \%(F(1,29)=23.02$, $P<.001)$. 

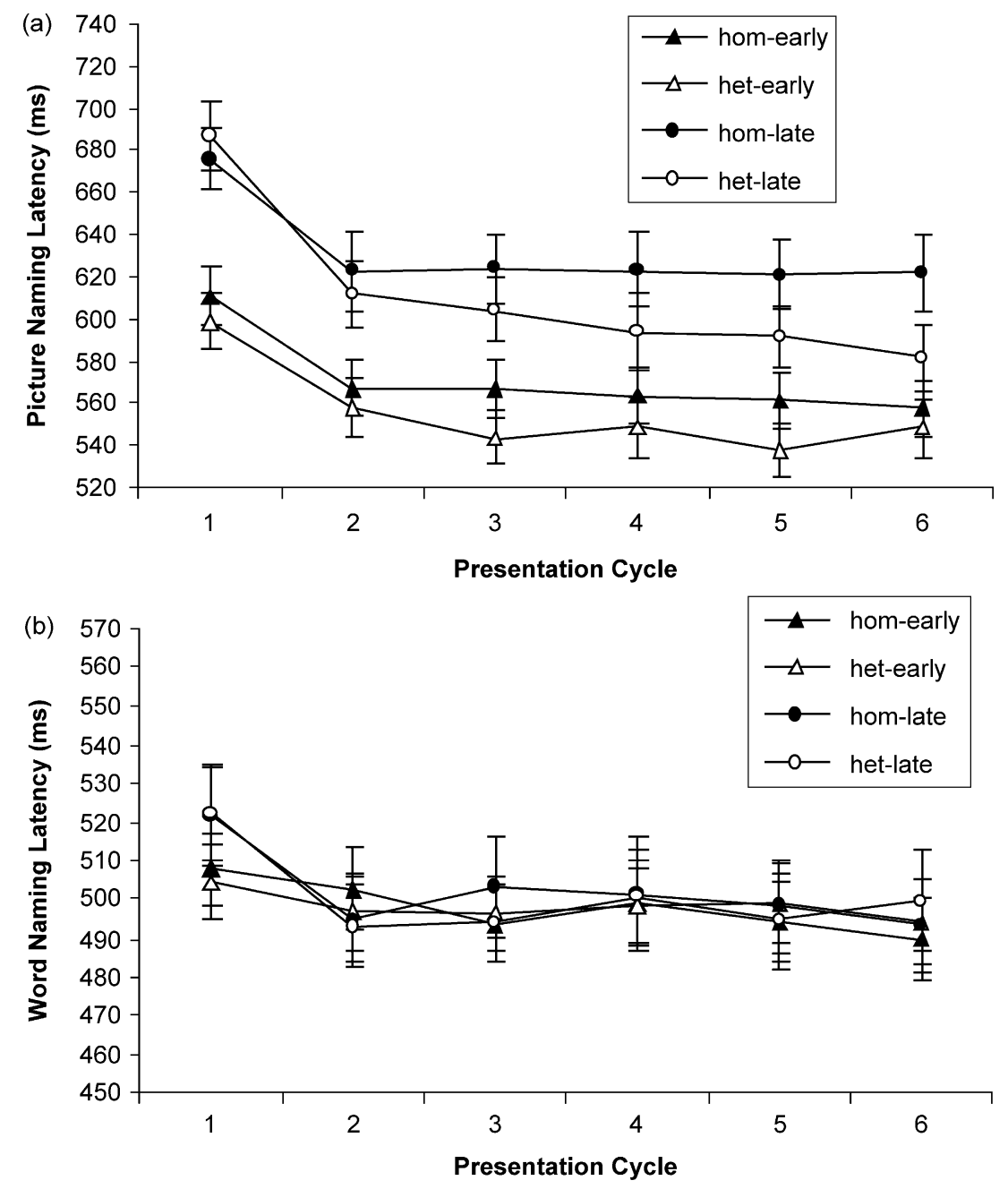

Fig. 1. Mean naming latencies by context (homogeneous (hom) vs. heterogeneous (het)), age-of-acquisition and presentation cycle in the picture naming (a) and the word naming (b) task. Error bars represent one standard error.

\subsection{Word naming}

The error rates were low (2.7\% overall) and did not differ across the experimental conditions. As Fig. 1(b) shows, the pattern of results obtained for the word naming latencies was quite different from the pattern seen for the picture naming latencies. In analyses of variance for the data from cycles two through six we obtained no significant main effect of any of the experimental variables or any significant interactions. When we entered AoA as the only predictor of reading latencies in a multiple regression analysis, 
it accounted for a mere $0.7 \%$ of variance. Adding word frequency to the list of predictors improved the fit significantly $\left(R^{2}=.17 ; F(1,29)=5.65, P<.05\right)$.

Supplementary analyses revealed a small effect of AoA $(16 \mathrm{~ms}, F(1,15)=5.07$, $P<.05)$ in the first presentation cycle. An AoA-effect of similar magnitude has been obtained before in word reading tasks (e.g. Gerhand \& Barry, 1998). The corresponding effect for picture naming was $77 \mathrm{~ms}(F(1,19)=85.46, P<.001)$ and significantly stronger than the effect obtained for word naming $(F(1,34)=30.32, P<.001)$. For cycles two through six we obtained a significant interaction of AoA and task $(F(1,34)=40.49$, $P<.001$ ), demonstrating that the AoA effect was much stronger for picture naming $(55 \mathrm{~ms})$ than for word naming $(2 \mathrm{~ms})$.

\section{Discussion}

Using a semantic blocking paradigm, we replicated the findings that (a) picture naming yielded a larger AoA-effect than word naming and (b) only pictures but not words were more difficult to name in a semantically homogeneous than in a heterogeneous context. Most importantly, we showed that in picture naming, the effect of semantic naming context - previously shown to arise at the lemma level - interacted with AoA-effects. As predicted, the context effect was stronger for late-acquired than for early-acquired object names. This interaction did not arise during the phonological encoding of the picture names, as it was not found when participants read the picture names aloud.

A potential concern is that the larger semantic context effect for the late-acquired than for the early-acquired pictures might be related to the longer naming latencies for the lateacquired set. To address this concern, we carried out a supplementary experiment with 16 participants, presenting the pictures in a degraded version, in which about $50 \%$ of their contours were deleted. Contrary to our expectation, the degradation did not much increase the overall naming latencies. However, we could combine the results from the present participants with those of the picture naming group described above and divide the resulting group into three groups of 12 persons each on the basis of their naming speed (fast, medium, slow).

Table 2 displays the results for the three groups of speakers. An ANOVA (including cycles two to six) confirmed significant main effects of speed group $(F(2,33)=38.29, P<$ $.001)$, AoA $(F(1,33)=107.98, P<.001)$ and semantic context $(F(1,33)=100.58, P<$ $.001)$. In line with Gerhand and Barry (1998), we also obtained a significant interaction between speed group and AoA $(F(2,33)=6.37, P=.005)$, with the magnitude of the AoAeffect increasing with the naming latencies. As expected, the interaction between AoA and semantic context was also significant $(F(1,33)=12.62, P<.001)$. Crucially, the three-way interaction of speed, AoA, and semantic context was not significant $(F<1)$ : All speed groups displayed larger context effects for late-than for early-acquired sets.

As explained in the Introduction, the semantic context effect arises during lemma selection but draws back to patterns of activation on the conceptual level. When all stimuli in a list come from the same semantic category, the corresponding lemmas activate each other via shared category and feature nodes, which renders the selection of a target lemma more time-consuming than when the stimuli stem from different 
Table 2

Mean picture naming latencies (ms, with standard errors) by AoA and semantic context and context effects for the fastest, the medium, and the slowest groups of participants

\begin{tabular}{|c|c|c|c|c|c|c|}
\hline \multirow[b]{2}{*}{ Speed Group } & \multirow[b]{2}{*}{ AoA } & \multicolumn{2}{|c|}{ Homogeneous Context } & \multicolumn{2}{|c|}{ Heterogeneous Context } & \multirow{2}{*}{$\begin{array}{l}\text { Context- } \\
\text { Effect }\end{array}$} \\
\hline & & M & SE & M & SE & \\
\hline \multirow[t]{2}{*}{ Fast } & Early & 526 & 11 & 510 & 12 & 16 \\
\hline & Late & 556 & 9 & 529 & 9 & 27 \\
\hline \multirow[t]{2}{*}{ Medium } & Early & 566 & 4 & 555 & 6 & 11 \\
\hline & Late & 632 & 4 & 599 & 4 & 33 \\
\hline \multirow[t]{2}{*}{ Slow } & Early & 636 & 15 & 616 & 13 & 20 \\
\hline & Late & 705 & 19 & 672 & 17 & 33 \\
\hline
\end{tabular}

semantic categories. For our current argument it is crucial that that the semantic context effect has been shown to generalise to new, not-yet-presented stimuli (Belke et al., in press). This suggests that the repeated selection of items from the same semantic category raises the activation levels of the target stimuli as well as the activation levels of other members of the same semantic category. We propose that the interaction of the effects of AoA and semantic context obtained in the present picture naming experiment arose because the selection of the lemmas of late acquired items was affected more strongly by the increased activation levels of extra-list competitors (many of which would be acquired earlier and would therefore be strong competitors) than the retrieval of the early acquired items. This account implies that the frequency-independent AoA effect observed in picture naming arises during lemma selection (Bates et al., 2001).

An important issue for further research is why lemmas of early acquired words are more potent competitors to other lemmas than lemmas of late acquired words. Steyvers and Tenenbaum (in press) presented a growing network model of the semantic system, in which new nodes connect to existing nodes as a function of how many connections the nodes already have (the more connections a node has, the more likely it is that new nodes will connect to it). Early-acquired lexical concept nodes have more central positions in the network than late acquired ones, and the associated lemmas will be co-activated faster and more intensively in word production than the lemmas associated with late-acquired concepts. This mechanism may be the origin of the difference in competitive strength between early- and late-acquired items at the lemma level.

The mapping between concepts and linguistic units (lemmas in our working model, P-lexemes in the model proposed by Caramazza, 1997), which is a requirement for picture naming, is entirely arbitrary. The mapping between orthographic and phonological representations, which is required for word naming, is comparatively regular. Thus, our allocation of the frequency-independent AoA effect at the level of lemma selection is compatible with the generalisation arising from a number of studies suggesting that AoA effects arise in tasks requiring arbitrary mappings between processing units (e.g. Bonin, Barry, Méot, \& Chalard, 2004; Ellis \& Lambon Ralph, 2000; Zevin and Seidenberg, 2002). 


\section{Acknowledgements}

We thank Victoria Jellett for testing most of the participants. This research was supported by a grant from the German Academic Exchange Service (DAAD; D/02/00789) to the first author.

\section{Appendix A}

Stimulus materials (homogeneous sets appear in rows, heterogeneous sets in columns)

\begin{tabular}{llll}
\hline $\begin{array}{l}\text { Early-acquired: } \\
\text { Orange }\end{array}$ & Banana & Pear & Carrot \\
Lion & Frog & Spider & Rabbit \\
Scissors & Paintbrush & Ladder & Hammer \\
Jumper & Trousers & Dress & Shoe \\
Late-acquired: & & & \\
Pepper & Cherry & Onion & Lettuce \\
Ostrich & Eagle & Beetle & Camel \\
Chisel & Pliers & Spanner & Broom \\
Tights & Shawl & Mitten & Waistcoat
\end{tabular}

\section{References}

Barry, C., Hirsh, K. W., Johnston, R. A., \& Williams, C. L. (2001). Age of acquisition, word frequency, and the locus of repetition priming in picture naming. Journal of Memory and Language, 44, 350-375.

Belke, E., Meyer, A. S., Damian, M. F. (in press). Refractory effects in picture naming as assessed in a semantic blocking paradigm. Quarterly Journal of Experimental Psychology: A.

Bates, E., Burani, C., D’Amico, S., \& Barca, L. (2001). Word reading and picture naming in Italian. Memory and Cognition, 29, 986-999.

Bonin, P., Barry, C., Méot, A., \& Chalard, M. (2004). The influence of age of acquisition in word reading and other tasks: A never ending story? Journal of Memory and Language, 50, 456-476.

Brown, G. D. A., \& Watson, F. L. (1987). First in, first out. Word learning age and spoken word frequency as predictors of word familiarity and word naming latency. Memory and Cognition, 15, 208-216.

Brysbaert, M., Ghyselinck, M. (in press). The effect of age-of-acquisition: Partly frequency-related, partly frequency-independent. Visual Cognition.

Brysbaert, M., Van Wijnendaele, I., \& De Deyne, S. (2000). Age-of-acquisition of words is a significant variable in semantic tasks. Acta Psychologica, 104, 215-226.

Caramazza, A. (1997). How many processing levels are there in lexical access? Cognitive Neuropsychology, 14, 177-208.

Coltheart, M., Rastle, K., Perry, C., Langdon, R., \& Ziegler, J. (2001). DRC: A dual route cascade model of visual word recognition and reading aloud. Psychological Review, 108, 204-256.

Damian, M. F., Vigliocco, G., \& Levelt, W. J. M. (2001). Effects of semantic context in the naming of pictures and words. Cognition, 81, B77-B86.

Dell, G. S., Schwartz, M. F., Martin, N., Saffran, E. M., \& Gagnon, D. A. (1997). Lexical access in aphasic and nonaphasic speakers. Psychological Review, 104, 801-838. 
Ellis, A. W., \& Lambon Ralph, M. A. (2000). Age of acquisition effects in adult lexical processing reflect loss of plasticity in maturing systems: Insights from connectionist networks. Journal of Experimental Psychology: Learning, Memory, and Cognition, 26, 1103-1123.

Gerhand, S., \& Barry, C. (1998). Word frequency effects in oral reading are not merely age-of-acquisition effects in disguise. Journal of Experimental Psychology: Learning, Memory, and Cognition, 24, 267-283.

Izura, C., \& Ellis, A. W. (2004). Age of acquisition effects in translation production tasks. Journal of Memory and Language, 50, 165-181.

Kroll, J. F., \& Stewart, E. (1994). Category interference in translation and picture naming: Evidence for asymmetric connections between bilingual memory representations. Journal of Memory and Language, 13, $149-174$.

Levelt, W. J. M., Roelofs, A., \& Meyer, A. S. (1999). A theory of lexical access in speech production. Behavioral and Brain Sciences, 22, 1-75.

Lewis, M. B., Gerhand, S., \& Ellis, H. D. (2001). Re-evaluating age-of-acquisition effects: Are they simply cumulative-frequency effects? Cognition, 78, 189-205.

Monaghan, J., \& Ellis, A. W. (2002). Age of acquisition and the completeness of phonological representations. Reading and Writing, 15, 759-788.

Morrison, C. M., Chappell, T. D., \& Ellis, A. W. (1997). Age of acquisition norms for a large set of object names and their relation to adult estimates and other variables. The Quarterly Journal of Experimental Psychology, $50 A, 528-559$.

Morrison, C. M., \& Ellis, A. W. (1995). Roles of word frequency and age of acquisition in word naming and lexical decision. Journal of Experimental Psychology: Learning, Memory, and Cognition, 21, 116-133.

Roelofs, A. (2004). Seriality of phonological encoding in naming objects and reading their names. Memory and Cognition, 32, 212-222.

Snodgrass, J. G., \& Vanderwart, M. (1980). A standardized set of 260 pictures: Norms for name agreement, image agreement, familiarity and visual complexity. Journal of Experimental Psychology: Human Learning and Memory, 6, 174-215.

Steyvers, M., Tenenbaum, J. (in press). Graph Theoretic Analyses of Semantic Networks: Small Worlds in Semantic Networks. Cognitive Science.

Zevin, J. D., \& Seidenberg, M. S. (2002). Age of Acquisition effects in word reading and othr tasks. Journal of Memory and Language, 47, 1-29. 